\title{
The Monitoring with Advanced Sensors, Transmission and E-Resuscitation in Traumatic Brain Injury (MASTER-TBI) collaborative: bringing data science to the ICU bedside
}

Robert McNamara, Shiv Meka, James Anstey, Daniel Fatovich, Luke Haseler, Melinda Fitzgerald and Andrew Udy

Moderate to severe traumatic brain injury (m-sTBI) is a highly complex disease ${ }^{1}$ with major public health costs and consequences. ${ }^{2-4}$ Unfortunately, despite much effort, there has not been any significant improvement in $\mathrm{m}$-sTBI clinical care and/or patient outcomes in the past 20 years. ${ }^{3,5}$ Major m-sTBI trials examining surgical decompression, 6,7 therapeutic hypothermia,, 89 osmotherapy, ${ }^{10}$ and intracranial pressure (ICP) monitoring ${ }^{11}$ have all failed to demonstrate an outcome benefit, suggesting that there is no single "magic bullet" for traumatic brain injury (TBI). In combination with improved understanding of TBI pathophysiology, ${ }^{1}$ this lack of improvement in patient outcomes calls into question whether continuing to apply a uniform approach ${ }^{12,13}$ to a complex, heterogeneous and dynamic condition ${ }^{1}$ remains optimal. In response, a developing modulated approach afforded by multimodal monitoring seeks to improve patient care and outcomes through the collection and analysis of highly granular data, which should allow for the individualisation of m-sTBI therapy. It is with this background in mind that in 2019 three metropolitan intensive care units (ICUs) near simultaneously adopted the use of ICM+ neuromonitoring software (Cambridge Enterprise, Cambridge, UK), a technology that allows for the capture and real time analysis of high resolution patient monitoring data. With the adoption of ICM+ software, a large amount of data generated in the process of caring for m-sTBI patients became available for further analysis. To facilitate data management and analysis, the units formed the MASTER-TBI collaborative in early 2020.

In simplest terms, the MASTER-TBI project involves the collation of digital data generated during the ICU treatment of m-sTBI patients. Data collected includes ICM+, medical record, medication, hospital record and/ or radiological data. Since gaining the requisite ethics and governance approvals in early 2020, the project has

\section{ABSTRACT}

Background: With the adoption of multimodal neuromonitoring techniques, a large amount of high resolution neurophysiological data is generated during the treatment of patients with moderate to severe traumatic brain injury (m-sTBI) that is available for further analysis. The Monitoring with Advanced Sensors, Transmission and E-Resuscitation in Traumatic Brain Injury (MASTER-TBI) collaborative was formed in 2020 to facilitate analysis of these data.

Objective: The MASTER-TBI collaborative curates m-sTBI patient data for the purposes of comparative effectiveness research, machine learning algorithm development, and neuropathophysiological phenomena analysis.

Design, setting and participants: The MASTER-TBI collaborative is a multicentre longitudinal cohort study which utilises a novel hybrid cloud platform and other data science-informed techniques to collect and analyse data from patients with m-sTBI in whom both intracranial pressure monitoring and $\mathrm{ICM}+$ (Cambridge Enterprise, Cambridge, UK) neuromonitoring software are utilised. MASTER-TBI enrols patients with m-sTBI from three participating Australian trauma intensive care units (ICUs).

Main outcome measures: Captured outcome measures available for analysis include pathophysiological events (intracranial hypertension, cerebral perfusion pressure variations etc), surgical interventions, ICU and hospital length of stay, patient discharge status, and, where available, Glasgow Outcome Score-Extended (GOS-E) at 6 months.

Results and conclusion: MASTER-TBI continues to develop data science-informed systems and techniques to maximise the use of captured high resolution m-sTBI patient neuromonitoring data. The highly innovative systems provide a world-class platform which aims to enhance the search for improved m-sTBI care and outcomes. This article provides an overview of the MASTER-TBI project's developed systems and techniques as well as a rationale for the approaches taken.

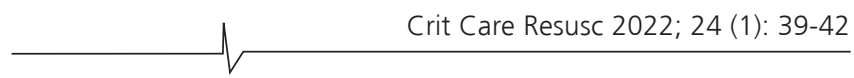




\section{ORIGINAL ARTICLES}

gathered files from about 200 patients. From the beginning, the project has focused on two interrelated activities: i) data collation and analysis, and ii) the development of data science-informed data handling systems. This latter activity, undertaken in cooperation with project data scientists, aims to bring data science and machine learning to the ICU bedside.

\section{Requirements for data science in the m-sTBI setting}

Data science, and in particular machine learning, is a discipline that will become a major part of clinical practice. ${ }^{14}$ To apply data science for the benefit of m-sTBI patients, several points need to be understood. In terms of data requirements, the training of any clinically relevant machine learning algorithm is dependent upon having available relevant outcome data. Examples of some of the outcomes captured by the MASTER-TBI collaboration include pathophysiological events (intracranial hypertension, cerebral perfusion pressure variations etc), surgical interventions, ICU and hospital length of stay, patient discharge status, and, where available, Glasgow Outcome Score-Extended (GOS-E) at 6 months. Algorithms at varying stages of development include those designed to predict intracranial hypertension events, ICU length of stay, and outcomes at hospital discharge and 6-months. For algorithm development and/or calibration, large data stores containing relevant processed data, termed "data warehouses", are necessary. The size and quality of the available data warehouse directly affects algorithm development and associated validation processes. In addition, for the adaptation of machine learning algorithms for the local environment, the availability of large, locally derived, and relevant data are a prerequisite. There are currently few available m-sTBI datasets, with the Collaborative European Neuro Trauma Effectiveness Research in TBI (CENTER-TBI) high resolution ICU cohort ${ }^{15}$ and the MIMIC-III databases ${ }^{16}$ being the best-known examples. As MASTER-TBI continues to collate and analyse data, it will develop into a data warehouse available for the training, validation and/or calibration of locally relevant m-sTBI machine learning algorithms. Crucial to this effort is the standardisation of data capture rates among units participating in the project (Table 1). The standardisation of capture frequencies allows for both conservation of data and efficient training of machine learning models when merging data files from different units or patients.

As mentioned, data warehouses are required for validation testing of machine learning algorithms. This is because in contrast to traditional clinical research, where effort is made to prevent errors through the undertaking of additional and/or larger clinical trials, to validate promising results, machine learning algorithm resilience is developed using large datasets. During the development of a machine learning algorithm, often several thousand analyses, data correlations and/or model iterations are performed. Consequently, erroneous results, measured in terms of precision and recall, are inevitable. To address this, validation testing is conducted to determine if an error adversely influencing machine learning algorithm performance has occurred. Internal validation commonly occurs using what is known as the "train, test rule", which splits the dataset into two unequal fractions. The larger of the two fractions is used for algorithm training, while the remaining smaller fraction is used for validation testing. Further, and more robust, external validation occurs where the algorithm is tested against other existing or new datasets. Once validated, periodic machine learning algorithm calibration using contemporaneous datasets is required to maintain algorithm accuracy in the face of clinical practice evolution.

In the overall effort to bring data science to the m-sTBI patient bedside, machine learning algorithm development is one part of the overall challenge. Systems to securely stream data to an operational algorithm for near realtime processing are required. In addition, as artefacts are unavoidable in the clinical setting, the system and/or the algorithm must be able to process artefact-containing data. To stream patient data for machine learning algorithm processing MASTER-TBI has developed multiple specialised systems. The first operational system involves the streaming of neuromonitoring data to a hybrid-cloud facility for artefact processing. High resolution wave data are preferentially used over numeric or time-averaged data due to wave data's inherently higher data density and other spectral properties (Table 2). The use of a secure hybrid-cloud facility has the advantages of being scalable, possessing a large computational capacity, and having reduced data latency in comparison to traditional serverbased approaches. In addition, the approach allows for integration of additional data sources, such as radiology and pathology investigations, and other forms of digital

Table 1. MASTER-TBI wave capture frequencies

\begin{tabular}{lc} 
Wave & Capture frequency \\
\hline $\mathrm{ECG}$ & $500 \mathrm{~Hz}$ \\
$\mathrm{ABP}, \mathrm{ICP}, \mathrm{CPP}, \mathrm{CVP}$ & $250 \mathrm{~Hz}$ \\
$\mathrm{SpO}_{2}, \mathrm{~T}, \mathrm{PbtO}_{2}$ & $1 \mathrm{~Hz}$ \\
\hline
\end{tabular}

$\mathrm{ABP}=$ arterial blood pressure; $\mathrm{CPP}=$ cerebral perfusion pressure; $\mathrm{CVP}=$ central venous pressure; $\mathrm{ECG}=$ electrocardiogram; $\mathrm{ICP}=$ intracranial pressure; $\mathrm{PbtO}_{2}=$ brain tissue oxygenation; $\mathrm{SpO}_{2}=$ oxygen saturation measured by pulse oximetry; $\mathrm{T}=$ temperature. 
Table 2. Rationale for capturing and storing health data as waves for the purposes of machine learning

\begin{tabular}{ll} 
Rationale & Explanation \\
\hline $\begin{array}{l}\text { Conservation of data } \\
\text { Algorithm accuracy }\end{array}$ & $\begin{array}{l}\text { Conversion of wave data to low resolution statistical data is irreversible } \\
\text { Machine learning algorithms can discover patterns from multimodal wave data which are not present in the } \\
\text { corresponding averaged or lower resolution data17 }\end{array}$ \\
$\begin{array}{l}\text { Computational } \\
\text { efficiency }\end{array}$ & $\begin{array}{l}\text { Waves are commonly the form by which data are captured from patients in the acute setting. Development } \\
\text { of machine learning algorithms using waves reduces the need for pre-processing and make wave-trained } \\
\text { algorithms inherently faster and more computationally efficient in comparison to those trained with averaged } \\
\text { data }\end{array}$ \\
\hline
\end{tabular}

clinical data that may not be available on a particular server. Another feature of the hybrid-cloud facility is the automated visualisation and advanced data analytical capabilities that are made possible by using a cloud-based time series database which stores, analyses and intelligibly presents m-sTBI patient wave data in real time.

\section{Bringing data science to the bedside}

MASTER-TBI's developing collaborative platform will provide a tool for future m-sTBI research in Australia. The scalable hybrid-cloud platform provides for a continuous iterative process to capture and analyse highly granular m-sTBI data, allowing for a deeper understanding of the treatments and neurophysiological burdens experienced by m-sTBI patients. The availability of cloud computing resources greatly enhances the computational power available to the project, which is particularly of relevance to machine learning algorithm development activities. MASTER-TBI's expansion to include additional neurotrauma ICUs through the adoption of ICM+ software will accelerate the development of an m-sTBI data warehouse that is representative of the region and its patients. As the cloud system is scalable and theoretically accessible globally, it provides the potential to deliver real time m-sTBI data collation, analysis, and machine learning algorithm processing capabilities on a national, regional and/or global scale.

Pragmatically, the platform provides participating sites with a facility for accessing novel machine learning algorithms, and for collating and/or cleaning both ICM+ and other monitor-derived data. With collection of highly granular data from multiple ICUs in the same manner, iterative assessment of either unintentional or intentional differences (through crossover trials) in unit practices promises to yield valuable information from both a research and quality control perspective. In addition, as has been demonstrated by multiple groups, the wealth of high resolution neurophysiological and neuropathophysiological data contained in the database lends itself to brain physics research, such as analysis of cerebral autoregulation and/or neuro-cardiac interactions.

\section{Conclusion}

The development of a highly innovative and novel data science-guided approach to m-sTBI clinical research is promising, with the main aims being to utilise existing highly granular bedside data to develop machine learning algorithms that accurately forecast physiological deterioration and ICU, hospital, and 6-month patient-centred outcomes. Moreover, by using cloud-based infrastructure, real-time data visualisation and feedback to clinical staff are possible. By combining these advances with the recently funded PRECISION-TBI project (Promoting evidence-based, data driven care for critically ill m-sTBI patients) (Medical Research Future Fund [MRFF] GNT2007705), which aims to establish a neurocritical care collaborative across all neurotrauma centres in Australia, the development of a world class $\mathrm{m}$-sTBI research platform and data warehouse can be realised. In doing so, the MASTER-TBI collaborative will allow us to bring data science to the bedside, and greatly enhance the search for improved m-sTBI clinical care and outcomes.

\section{Competing interests}

All authors declare that they do not have any potential conflict of interest in relation to this manuscript.

\section{Author details}

Robert McNamara ${ }^{1,2}$

Shiv Meka ${ }^{3}$

James Anstey ${ }^{4}$

Daniel Fatovich ${ }^{5,6}$

Luke Haseler ${ }^{7}$

Melinda Fitzgerald 7,8

Andrew Udy ${ }^{9,10}$

1 Department of Intensive Care Medicine, Royal Perth Hospital, Perth, WA, Australia.

2 School of Medicine, Curtin University, Perth, WA, Australia.

3 Data Innovation Laboratory, Western Australian Department of Health, Perth, WA, Australia. 


\section{ORIGINAL ARTICLES}

4 Department of Intensive Care, Royal Melbourne Hospital, Melbourne, VIC, Australia.

5 Emergency Medicine, Royal Perth Hospital, University of Western Australia, Perth, WA, Australia.

6 Centre for Clinical Research in Emergency Medicine, Harry Perkins Institute of Medical Research, Perth, WA, Australia.

7 Curtin Health Innovation Research Institute, Curtin University, Perth, WA, Australia.

8 Perron Institute for Neurological and Translational Sciences, Perth, WA, Australia.

9 Australian and New Zealand Intensive Care Research Centre, School of Public Health and Preventive Medicine, Monash University, Melbourne, VIC, Australia.

10 Department of Intensive Care, Alfred Health, Melbourne, VIC, Australia.

Correspondence: Robert.mcnamara@health.wa.gov.au

doi: https://doi.org/10.51893/2022.1.OA5

\section{References}

1 Marklund N, Tenovuo O. Pathophysiology of severe traumatic brain injury. In: Sundstrøm T, Grände PO, Luoto T, et al; editors. Management of severe traumatic brain injury: evidence, tricks, and pitfalls. Springer, 2020; pp 35-50.

2 Taylor CA, Bell JM, Breiding MJ, Xu L. Traumatic brain injuryrelated emergency department visits, hospitalizations, and deaths - United States, 2007 and 2013. MMWR Surveill Summ 2017; 66: 1-16.

3 Brazinova A, Rehorcikova V, Taylor MS, et al. Epidemiology of traumatic brain injury in Europe: a living systematic review. J Neurotrauma 2021; 38: 1411-40.

4 Quaglio G, Gallucci M, Brand H, et al. Traumatic brain injury: a priority for public health policy. Lancet Neurol 2017; 16: 951-2.

5 Toida C, Muguruma T, Gakumazawa M, et al. Age- and severityrelated in-hospital mortality trends and risks of severe traumatic brain injury in Japan: a nationwide 10-year retrospective study. J Clin Med 2021; 10: 1072.

6 Cooper DJ, Rosenfeld JV, Murray L, et al. Decompressive craniectomy in diffuse traumatic brain injury. $N$ Engl J Med 2011; 364: 1493-502.
7 Hutchinson PJ, Kolias AG, Timofeev IS, et al. Trial of Decompressive craniectomy for traumatic intracranial hypertension. N Engl J Med 2016; 375: 1119-30.

8 Andrews PJ, Sinclair HL, Rodriguez A, et al. Hypothermia for intracranial hypertension after traumatic brain injury. N Engl J Med 2015; 373: 2403-12.

9 Cooper DJ, Nichol AD, Bailey M, et al; POLAR Trial Investigators and the ANZICS Clinical Trials Group. Effect of early sustained prophylactic hypothermia on neurologic outcomes among patients with severe traumatic brain injury: the POLAR randomized clinical trial. JAMA 2018; 320: 2211-20.

10 Roquilly A, Moyer JD, Huet O, et al; Atlanrea Study Group and the Société Française d'Anesthésie Réanimation Research Network. Effect of continuous infusion of hypertonic saline vs standard care on 6-month neurological outcomes in patients with traumatic brain injury: the $C O B I$ randomized clinical trial. JAMA 2021; 325: 2056-66.

11 Chesnut RM, Temkin N, Carney N, et al; Global Neurotrauma Research Group. A trial of intracranial-pressure monitoring in traumatic brain injury. N Engl J Med 2012; 367: 2471-81.

12 Carney N, Totten AM, O'Reilly C, et al. Guidelines for the management of severe traumatic brain injury, fourth edition. Neurosurgery 2017; 80: 6-15.

13 Hawryluk GWJ, Aguilera S, Buki A, et al. A management algorithm for patients with intracranial pressure monitoring: the Seattle International Severe Traumatic Brain Injury Consensus Conference (SIBICC). Intensive Care Med 2019; 45: 1783-94.

14 van de Sande D, van Genderen ME, Huiskens J, et al. Moving from bytes to bedside: a systematic review on the use of artificial intelligence in the intensive care unit. Intensive Care Med 2021; 47: 750-60.

15 Maas AIR, Menon DK, Steyerberg EW, Cet al; CENTRETBI Participants and Investigators. Collaborative European NeuroTrauma Effectiveness Research in Traumatic Brain Injury (CENTER-TBI): a prospective longitudinal observational study. Neurosurgery 2015; 76: 67-80.

16 Johnson AE, Pollard TJ, Shen L, et al. MIMIC-III, a freely accessible critical care database. Sci Data 2016; 3: 160035.

17 Hüser M, Kündig A, Karlen W, et al. Forecasting intracranial hypertension using multi-scale waveform metrics. Physiol Meas 2020; 41: 014001. 